\title{
Patient-Identified Markers of Quality Care: Improving HIV Service Delivery for Older African Americans
}

\author{
Brandon D. Mitchell ${ }^{1}$ (D . Liz Utterback ${ }^{1} \cdot$ Paul Hibbeler $^{1} \cdot$ Ashley R. Logsdon ${ }^{1} \cdot$ Patricia F. Smith ${ }^{1} \cdot$ Lesley M. Harris $^{1}$. \\ Billie Castle ${ }^{2} \cdot$ Jelani Kerr ${ }^{2} \cdot$ Timothy N. Crawford ${ }^{3}$
}

Received: 23 June 2021 / Revised: 11 January 2022 / Accepted: 12 January 2022 / Published online: 21 January 2022

(c) W. Montague Cobb-NMA Health Institute 2022

\begin{abstract}
Background Over 50\% of new AIDS/HIV diagnoses are older adults and disproportionately African American people. Longstanding health inequities, driven by the enduring nature of systemic racism, pose challenges to obtaining optimal HIV services. Patient experiences and identities shape the health care experience, yet patient voices are often minimized, including their assessment of quality HIV care. Understanding these markers of care, including facilitators of and barriers to care and engagement, may help enhance the patient voice, potentially improving service delivery and eradicating HIV healthcare disparities.

Method Using a convergent mixed method design, our study identifies patient-identified markers of quality care among older African Americans $(N=35)$. Measurements of global stress, HIV stigma, and engagement in care were collected, and in-depth qualitative interviews explored the symbols of quality care as well as facilitators of and barriers to care.

Results We identified widespread participant awareness and recognition of quality care, the detection of facilitators and barriers across individual, clinic, and community levels. Facilitators of care include diet, health, relationships, community support, and compassionate HIV care. Barriers to care include health comorbidities, economic, food, and housing insecurity, lack of transportation, and structural racism.

Conclusion Our findings illuminate how the prominence of barriers to care often uproot facilitators of care, creating impediments to HIV service delivery as patients transition through the HIV care continuum. We offer implications for practice and policy, as well as recommendations for reducing structural barriers to care by enhancing the patient voice and for aligning services toward compassionate and inclusive care.
\end{abstract}

Keywords AIDS/HIV · Older adults · Quality care · Patient voice · African American · Qualitative study · Critical race theory

Over the past few decades, breakthroughs in HIV/AIDS research have resulted in advances in treatment and preventive care [1]. As treatment and diagnostic detection improve, the number of people who are aging with HIV is also increasing. The Centers for Disease and Control and Prevention $(\mathrm{CDC})$ stated that one in every six newly diagnosed

Brandon D. Mitchell

brandon.mitchell@louisville.edu

1 Kent School of Social Work, University of Louisville, Louisville, KY, USA

2 School of Public Health and Information Sciences, University of Louisville, Louisville, KY, USA

3 Family Medicine and Population and Public Health Sciences, Wright State University, Dayton, OH, USA persons living with HIV is over the age of 50 [2]. Persons of older age are not tested as often and maybe less likely to consider testing, leading to diagnostic and treatment barriers [3]. Furthermore, the CDC estimates that $71 \%$ of HIV-positive older adults received some HIV care, 57\% were retained in care, and $64 \%$ were virally suppressed. However, less is known about how older adults experience their HIV care or what they consider to be high-quality HIV care [2].

Upon disaggregating HIV prevalence, health inequities are noted across diagnostic rates, quality of care, and viral suppression. For instance, African American people disproportionately comprise $42 \%$ of all HIV diagnoses, with disparate rates continuing across older populations [4]. Additionally, an array of barriers to care exist outside of the HIV health system, including economic, social, and behavioral 
ramifications often stemming from systemic racism [4-6]. The ramifications are illuminated by access and engagement barriers to care. For instance, transportation barriers and economic challenges have been shown to negatively impact health care access [7, 8]. Linkage to and engagement in care are further complicated by housing and food insecurity [9]. Environmental barriers to care are wide-ranging and may intersect with mental health challenges, as well as stress, stigma, substance use problems, and health comorbidities $[10,11]$.

The COVID-19 pandemic adds to the long list of barriers to HIV care and service provision and has exacerbated preexisting racial health inequities [12]. The pandemic has impacted testing capabilities, linkage to HIV care, and maintenance in care, with these barriers likely being more pronounced for populations who experience marginalization [13]. Coinciding with the pandemic has been growing recognition of the ongoing police violence against African American people, underscoring a need for new knowledge and efforts toward health equity [14]. One avenue to help support this transition toward health equity is to center the voices of older African American people across the HIV care continuum $[9,13]$. Older African American people living with HIV possess important historical experiences, values, and goals that are useful to understand and incorporate into their healthcare experiences $[9,15]$. This community is uniquely positioned at the intersection of multiple marginalized identities and is therefore instrumental in helping healthcare providers understand these patients' individual needs, improve services, and reduce barriers to care [13-20].

Given the depth of the challenges preventing African American people living with HIV from accessing, engaging, and remaining in care, it is important to offer an inclusive medical care model that centers the patient's voice in treatment $[16,21-28]$. The hierarchical structure of healthcare settings often creates additional forms of stigma and discrimination that are overtly or unconsciously reinforced by healthcare providers $[9,10,27,28]$. Recently, scholars have paid increased attention to the patient's role in facilitating engagement in care $[9,10,16,21-28]$. Outside of healthcare settings, many individuals receive support from loved ones and deploy coping and resiliency strategies to help manage their HIV care in a unique and strategic manner [27-29]. With populations who have experienced historical marginalization, it is imperative that healthcare providers understand the patient's experiences and knowledge in ways that encourage improved health outcomes $[9,10]$.

Enhancing the patient voice in HIV healthcare can be achieved through a number of different advancements in care, including patient-centered, patient navigation, strengthening patient-provider relationships, compassionate care, and through the utility of practices such as critical race theory and anti-racist strategies. These strategies may offer the potential to ameliorate HIV disparities, ultimately improving healthcare equity. Ryan White has played a central role in pioneering patient-centered approaches, offering inclusive support services well beyond the healthcare setting [30]. A complete review of patient-centered trends is beyond the scope of this paper, however, we aim to support this paradigm shift by highlighting several components that may be instrumental to enhancing the patient voice and bolstering care.

Efforts to enhance the patient voice may be an effective avenue to reduce HIV disparities if conjoined by four strategic support levels: quality care, relationships, compassion, and structural/equity-based. First, amplifying the patient voice must be supported by quality HIV care that includes concurrent compassionate-based frameworks and not a replacement of medical supervision [31]. Second, supportive, healthy, loving relationships between providers and patients can be emphasized within patient-centered healthcare practices. Relationship-focused healthcare is the cornerstone of patient-navigation trends, which monitor and facilitate ideal healthcare progression [32-34]. Furthermore, relationship promotion for aging populations has been shown to enhance the quality of life, offering increases in patient self-efficacy and resiliency [9, 31, 34-38]. Third, compassionate-based care can be achieved through bolstering the patient voice, while offering a host of physiological and psychological benefits [31]. Compassion has been identified as a protective factor throughout the medical field and offers a road map to help reduce isolation, improve engagement in care, strengthen self-care, build trust, reduce pain, and increase life expectancy [31]. Furthermore, components of compassion, including care and empathy, have been shown to improve quality care and adherence, reduce viral load, and may simultaneously stimulate benefits to healthcare providers $[31,39,40]$. Fourth, centering the patient voice may help promote a structural focus and facilitate equitable practices. Anti-racist movements in healthcare, critical race theory, and intersectionality offer great utility to promote patientcentered care and help to realign focus toward disparity reductions and healthcare equity [14, 41, 42]. Collectively, these levels of patient-centered support offer a sound path toward building capacity, strengthening an understanding of patient experiences in care, and building support for an older African American population living with HIV [9, 31-43]

As the world recovers from the COVID-19 pandemic, we must simultaneously recover from the pandemic of racism present within the healthcare system, in part by restoring trust in the HIV care medical model and treatment. The aim of this study is to explore African American patient voices across the HIV healthcare continuum. We propose that a greater understanding of patient-identified markers of quality care may improve HIV care. We situate our study within the context of two overarching research questions: 
(1) What are the patient-identified markers of quality care, facilitators of care, and barriers to care for older African Americans living with HIV?

(2) From the perspective of older African Americans living with HIV, how do medical providers prioritize patientidentified markers of quality care in healthcare services throughout the HIV care continuum?

\section{Materials and Method}

This study deployed a convergent mixed methods design, utilizing a variety of data sources, including surveys, interviews, and focus groups to explore the healthcare experiences of older African Americans living with HIV/AIDS [44]. Within this design, the qualitative research was informed by constructivist grounded theory situated within a theoretical framework of symbolic interactionism [45, 46]. The tenets of symbolic interactionism were used to help identify the symbols of quality care and the symbolic barriers to care, among other important experiences, as identified by study participants [47]. Our convergent design integrated quantitative and qualitative data by concurrently analyzing different types of data [44].

\section{Study Population}

Thirty-five participants from a major midwestern metropolitan city who were living with HIV, over the age of 50, and identified as African American participated in this study.

\section{Data Collection}

Participant selection was established through flyers posted at HIV clinics. All participants $(N=35)$ provided consent and were fluent English speakers. Participants completed the Berger HIV Stigma Scale, Perceived Stress Scale, Engagement with Health Care Providers Scale, and a Composite of Engagement in HIV Care Scale. Researchers conducted indepth qualitative interviews with all thirty-five participants and one final focus group. The focus group was designed to provide rich informant feedback. Six participants with differential scores on the self-rating stress, stigma, and engagement in care scales were selected for focus groups, thereby representing various experiences. Upon completion of the qualitative interview and survey process, participants were provided with a $\$ 35$ gift card, and the members of the focus group received an additional $\$ 25$ gift card. To maintain informed consent and data privacy of all participants, all study participants used pseudonyms during the interview sessions.

\section{Measures}

The Berger HIV Stigma Scale entails 40 self-rated items across a 4-point scale ranging from "strongly agree" to "strongly disagree." All items are summed to create a composite stigma score, categorized in our study as low, medium, or high. The Berger HIV Stigma Scale received a Cronbach's alpha of 0.98 , evidence of excellent internal reliability [48]. The Perceived Stress Scale assesses levels of stress with scores ranging from 0 to 40 , where higher scores indicate more stress: a score of $0-13$ is categorized as low stress, $14-26$ as moderate, and $>26$ as high. Reliability was previously assessed using Cronbach's alpha, with scores ranging between 0.84 and 0.86 , indicative of good internal consistency [49]. The Engagement in HIV Care scale consists of seven questions regarding appointment engagement, medical knowledge, and antiretroviral adherence (ARV). The seven items are summed to assess the overall engagement level across three levels ranging from 0 to 7 $(0-4=$ low engagement, $5-6=$ moderate engagement, and $7=$ high engagement) [50].

\section{Data Analysis}

Both quantitative and qualitative data were analyzed and integrated using Dedoose 8.3.43 analytical software. Quantitative data included descriptive reporting for continuous and categorical data, meanwhile, Cronbach's alpha was used to assess internal consistency across all study scales. Dedoose software was also used to integrate quantitative and qualitative data (e.g., scale descriptors and qualitative coding heat map).

Constructivist grounded theory strategies guided the analysis of the qualitative data [44]. First, interview transcripts were coded using line-by-line data analysis techniques by applying gerunds (nouns ending in -ing) to account for action within the participants' narratives. We then clustered our codes into focused codes using an iterative analytical process to develop our study's codebook. Our final codebook consisted of 24 codes with full definitions, which were uploaded to Dedoose analytical software to support our coding and analysis. Using Dedoose, we coded 11 transcripts and assessed interrater reliability statistics using Cohen's Kappa test. In this pooled analysis, Cohen's $k=0.81$, indicated substantial coding agreement across researchers. After iterative processing and discussion, we were able to resolve disagreements. Reassessing interrater reliability statistics generated Cohen's $k=0.98$, indicating excellent agreement. Finally, peer debriefing and consensus building led to minor inclusionary adjustments to the codebook; we then proceeded to code the remaining 24 interview transcripts and one focus group transcript. 
Integration of quantitative and qualitative data was facilitated by the creation of a data matrix [51]. This process enabled the research team to understand the linkages between the stress, stigma, and engagement in care scores and the absence or presence of narratives related to barriers and facilitators of quality HIV care (see Supplemental Table 1).

\section{Results}

\section{Sample Characteristics}

Thirty-five participants were eligible and agreed to participate in the study. The mean age was $58.1 \pm 5.2$ years; $42.3 \%$ were $\geq 60$ years old. Most of the participants were male (63\%), had some college education (59.3\%), and identified as heterosexual (48.2\%). Fifty-nine (59.3\%) of participants had a history of incarceration and substance use, while nearly half $(46.2 \%)$ had a history of homelessness. Participants also reported the duration of their HIV was almost one-third of the mean age (21.2 \pm 7.4 years). For complete demographic characteristics, see Table 1 .

\section{Stress, Stigma, and Engagement in Care Scales}

All scales had acceptable values $(>0.80)$ except for the Engagement in Care Scale (0.40). The mean Engagement in Care Scale score was 5.2 \pm 1.4 , and $54.3 \%$ were considered moderately engaged in care. The overall stigma score was high, with a mean of $97.7 \pm 23.9$, and most participants were moderately stressed, with a mean of $18.9 \pm 8.4$. Cronbach's alphas for the study variables are presented below in Table 2 .

\section{Integrated Findings}

The combination of our quantitative and qualitative analysis of the experiences of older African Americans living with HIV led to the creation of two overarching categories: (1) facilitators of care and (2) barriers to care. As illustrated in Fig. 1, facilitators of care are presented across three domains of the clinic, community, and individual levels. Barriers to care disrupted the facilitators of care, also presented across clinic, community, and individual levels. In this section, we support our qualitative analysis with convergent and divergent themes related to our quantitative scales of interest (i.e., engagement, stress, and stigma). For instance, $68 \%$ of study participants reported barriers within the clinic, $48 \%$ reported individual or community level barriers, and $71 \%$ reported structural level barriers to care (Supplemental Table 1; Fig. 1). Facilitators to care were noted by participants in similar prominence. Specifically, $71 \%$ of participants reported clinic level facilitators to care, $68 \%$ individual or community level facilitators, and $60 \%$ noted support as a facilitator to
Table 1 Demographic and clinical characteristics among older African American adults living with HIV $(N=35)$

\begin{tabular}{|c|c|}
\hline & $n(\%)$ \\
\hline Age, mean (std) & $58.3(5.4)$ \\
\hline $50-54$ years & $10(29.4)$ \\
\hline $55-59$ years & $10(29.4)$ \\
\hline$\geq 60$ years & $14(41.2)$ \\
\hline \multicolumn{2}{|l|}{ Gender identity } \\
\hline Cisgender man & $24(68.6)$ \\
\hline Cisgender woman & $9(25.7)$ \\
\hline Transwoman & $2(5.7)$ \\
\hline \multicolumn{2}{|l|}{ Education } \\
\hline High school degree or less & $10(28.6)$ \\
\hline Some college & $18(51.4)$ \\
\hline College degree & $7(20.0)$ \\
\hline \multicolumn{2}{|l|}{ Sexual orientation } \\
\hline Heterosexual & $18(51.4)$ \\
\hline LGB or questioning & $17(48.6)$ \\
\hline \multicolumn{2}{|l|}{ Relationship status } \\
\hline Single & $17(48.6)$ \\
\hline In a relationship & $5(14.3)$ \\
\hline Divorced/separated & $13(37.1)$ \\
\hline \multicolumn{2}{|l|}{ Employment status } \\
\hline Employed & $11(32.4)$ \\
\hline Unemployed & $6(17.7)$ \\
\hline Retired/unable to work & $17(50.0)$ \\
\hline Insurance-Yes & $31(88.6)$ \\
\hline History of homelessness-Yes & $17(50.0)$ \\
\hline History of substance use-Yes & $23(65.7)$ \\
\hline AIDS diagnosis-Yes & $13(37.1)$ \\
\hline On antiretroviral medication-Yes & $32(94.1)$ \\
\hline $\begin{array}{l}\text { Missed any clinic appointments in the past } 6 \text { months- } \\
\text { Yes }\end{array}$ & 7 (20.6) \\
\hline $\begin{array}{l}\text { Missed any clinic appointments in the past } 12 \text { months- } \\
\text { Yes }\end{array}$ & $21(61.8)$ \\
\hline Years living with HIV_-mean years (std) & $20.8(8.0)$ \\
\hline
\end{tabular}

Missing values are not included in calculation of percentages

care (Supplemental Table 1; Fig. 1). Even amidst substantial barriers to care, participants were able to recognize and value the facilitators to care when they were experienced or received in the clinic. These findings are expounded upon throughout the following section.

\section{Category 1: Facilitators of Care}

Many participants identified positive experiences in care that facilitated their engagement, improved outlooks on living with HIV, and enhanced feelings of support and well-being. Herein, we present facilitators of care across the clinic, community, and individual levels. 
Table 2 Stress, stigma, and engagement in care scale means

\begin{tabular}{llll}
\hline Scale & Mean (SD) & $95 \%$ CI & Cronbach $\alpha$ \\
\hline Perceived stress scale & $18.9(8.4)$ & $16.0-21.8$ & 0.80 \\
HIV-related stigma scale & & & \\
$\quad$ Overall stigma & $97.7(23.9)$ & $89.4-105.9$ & 0.96 \\
$\quad$ Personalized stigma & $42.2(12.7)$ & $37.8-46.5$ & 0.95 \\
$\quad$ Disclosure stigma & $27.7(6.2)$ & $25.6-29.8$ & 0.86 \\
$\quad$ Negative self-image stigma & $28.5(8.3)$ & $25.7-31.4$ & 0.88 \\
$\quad$ Public attitudes stigma & $49.6(13.3)$ & $45.0-54.2$ & 0.95 \\
Engagement with healthcare providers & $15.5(4.7)$ & $13.8-17.1$ & 0.94 \\
Engagement in care & $5.2(1.4)$ & $4.7-5.7$ & 0.40 \\
$\quad$ Low engagement, $n(\%)$ & $9(25.7)$ & & \\
$\quad$ Moderate engagement, $n(\%)$ & $19(54.3)$ & & \\
$\quad$ High engagement, $n(\%)$ & $7(20.0)$ & & \\
\hline
\end{tabular}

Fig. 1 The patient experience of older African Americans living with HIV

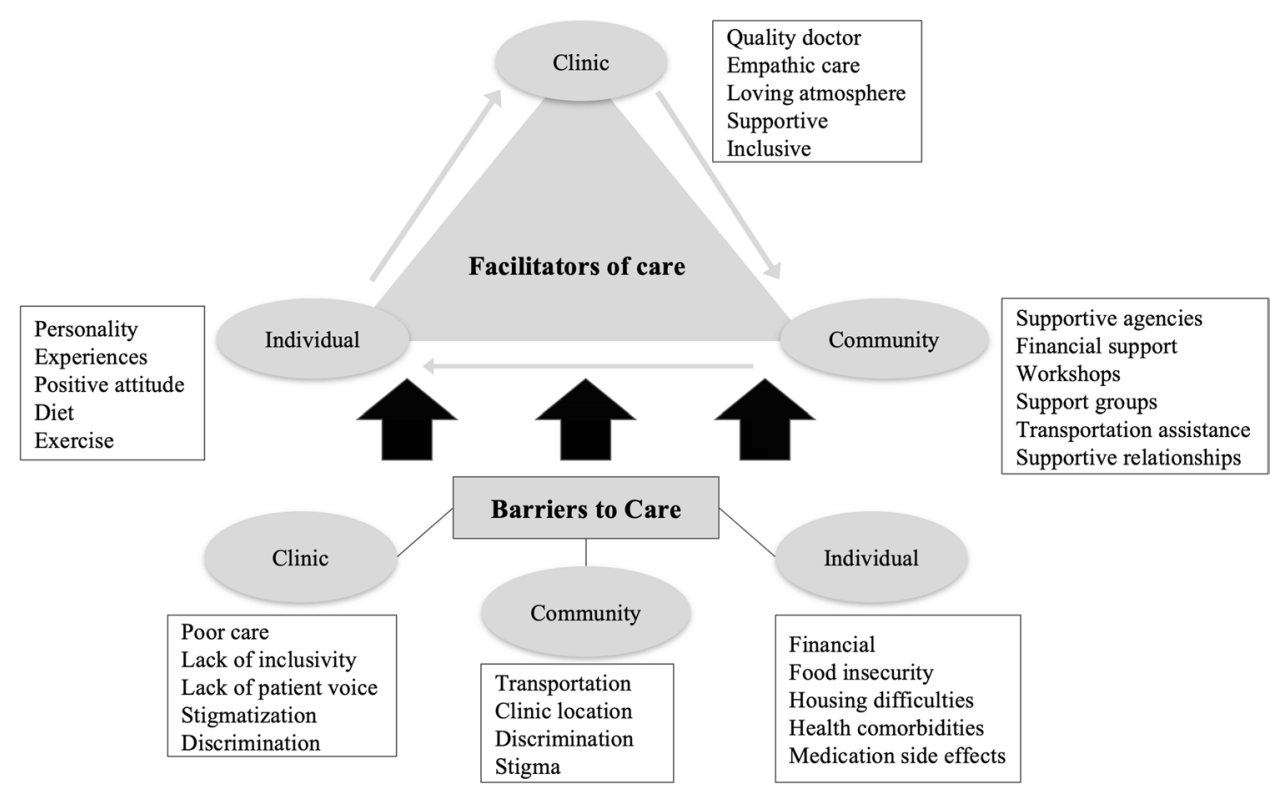

Clinic Facilitators of Care Within the clinic, participants shared their appreciation for quality medical experiences, including empathetic physicians and a caring atmosphere that spurred their future engagement. Through patient education, participants learned more about their diagnosis, thus feeling empowered to stay engaged in care. "Topher" voiced concerns over the extent of medication side effects, but through conversations with his doctor, he was able to understand the importance of medication adherence. Through experience and key health expertise, Topher was able to recognize that, in the long run, the medication would be instrumental to his prolonged health and survival. Topher described his conversation with his infectious disease physician, who told him, "If you get off the medication, initially you're going to feel better because you don't have all those toxins from the medication. But further down the road, you're going to either see me or the coroner." Topher expressed appreciation for the direct way his physician delivered this news with concern and compassion for him as the patient. This message was instrumental to Topher's future engagement in care. It is important to note that the doctor's comment was made in combination with a thorough medical exam and appropriate testing-as Topher stated, "a head-totoe appraisal." Topher mentioned the doctor by name multiple times during the interview, describing the vital role she played in his life by saying, "Dr. $\mathrm{X}$ was a lifesaver. If anybody is going to be canonized, it should be her; she did really good." Finally, Topher reported moderate engagement in care, medium stigma, and low-stress levels, likely influenced by his quality care experiences.

Participants identified an array of symbolic markers of quality care that were instrumental to their long-term care. For instance, participants expressed tremendous appreciation of quality care when it was received. "Eminem" talked 
about feeling valued as a patient: "[the doctor] made me feel comfortable, and told me that I could live for a long time with HIV, as long as I did the right thing." Eminem's survey data showed high engagement in care, moderate stress, and medium levels of stigma, illuminating a clear relationship between feeling valued as a patient and HIV care engagement. "Rabbit" stated professionalism and friendliness as most valuable to him, however he reported low engagement, moderate stress, and medium stigma. It was unclear whether these valued traits were experienced throughout care. "Josephine" mentioned some empowering conversations with clinicians and mutual trust with HIV service providers, saying she felt loved and safe. Trust was so essential to her care that when her favorite doctor moved to a new clinic, she chose to go to the doctor's new facility. She reported low engagement, moderate stress, and high stigma, further situating the importance of compassionate care.

"Bo" reported moderate engagement and stress and medium stigma. He gave an example of an empathetic, loving environment that facilitated adequate care by stating, "For some reason, I just liked her bedside manner, you know? She used to talk to us and everything." "Greg" had moderate engagement and stress and medium stigma. Consistent with others, he discussed the importance of loving, supportive, and welcoming environments, which motivated him to maintain engagement in care. Similarly, "Beau" described the influence of providers' communication skills, attitude, and the benefits of inclusive and compassionate care, saying, "Her attitude, and when she come in there, she wouldn't come in there by talking at me. She would come in there like she was talking to me, smile on her face." Beau reported moderate engagement and stress and was one of the few to report low levels of stigma, a likely extension of the inclusive care experiences she reported. Collectively, patients had varying levels of engagement, stress, and stigma, yet many were able to recognize the markers of quality care, whether or not they were consistent experiences received.

Community Facilitators of Care Most participants cited community support, including agencies, workshops, and support groups that facilitated their longevity in care. "Doc" mentioned the importance of workshops and the benefits of establishing community support and relationships outside the HIV clinic. Even amidst low engagement, high stress, and stigma, Doc recognized the value of community-based support services. "Daphne" reported mid-levels of engagement, stress, and stigma and keenly alluded to communitybased agencies that she turned to for support, including workshops, education, and the overarching warmth and love received from these agencies. Daphne describes the services and the support she received:
I was given 20 tickets a month (for busing). They have a clothes closet. They have the ABC food pantry. They have different things that just really help you. They even have dentures; you get your teeth done. They have the RAND Foundation and stuff like that. They hook you up with organizations that work with people with HIV.

Many participants noted their gratitude for the supportive relationships within the community, while others spoke about loving relationships with their family members. "Bo" states, "I was literally floored, because of support." Participants also spoke about challenges due to HIV disclosure and the benefits of having a confidant to discuss their diagnosis with. "Tony" surrounded himself with supportive people and reported low stress and stigma. Others described the supportive recovery community, peer support groups, Alcoholics Anonymous meetings, church friends, and family members. "Nana" described his family members as "Supportive. My kids know and they call, and they ask, 'Daddy, how you doing? You all right? You taking your medicine? You need to come on down here and let us take care of you." This support translated into Nana maintaining moderate engagement, and mid-level reports of stress and stigma. "Jethro" listed an array of supportive community agencies, physicians, and family members: "My mother, for one. My sister, she passed of cancer a few years ago. My doctors and my nurse practitioner and most of the people down to the VA hospital. They have been very supportive of me. Without them, I probably wouldn't be here." Jethro was highly engaged in HIV care, however even amidst the wide-ranging support he experienced high stress and stigma. Consistent with other participants, quality care and community support were helpful, yet often did not alleviate high levels of stress and stigma.

Individual Facilitators of Care Several participants mentioned empowering perspectives, motivating personalities, and healthy lifestyle choices. Nana discussed how his mindset and optimism enabled him to establish and maintain care. "Gigi" described an array of coping strategies, including adhering to her medication regimen, regularly attending therapy and healthcare appointments, and maintaining long-term sobriety. Even amidst these effective strategies, Gigi reported low engagement in HIV care, moderate stress, and high levels of stigma. Topher said, "I'll do a little bit of exercise with my dumbbells, stretching and working out, or going for a walk every once in a while, and the yard work and staying active. Staying active is one thing, and watching what you eat. It really has a lot to do with it." There was a reoccurring theme of perseverance that enabled participants to feel motivated, however, an empowered outlook did not prevent participants from experiencing stress, stigma, and barriers to care. 


\section{Category 2: Barriers to Care}

Nearly every participant mentioned barriers to care in some form. This section delineates barriers noted by participants across the clinic, community, and individual levels.

Clinic Barriers to Care Participants identified barriers associated with the high cost of services, poor treatment from insurance agencies, and a lack of clinical support. Nana maintained moderate engagement in care even amidst her ongoing gaps in governmental health insurance:

Then I call in my Medicaid at the Clinic, she said, 'No, I don't need to contact you. Try to call up when you turn 65.' I said, 'Hell, I should've stayed 64.' Then the next thing is, I got a bunch of co-pays. I turn 65 and I'm supposed to be in my golden years and I'm losing benefits. I got to pay out more money than I did before I turned 65. It's ridiculous.

According to participants, the government systems designed to support and provide medical care are overly complex and increase out-of-pocket costs. Gigi echoed Nana's observations about medical copays: "It's just the differences in the different types of Medicaid now, because before Medicaid, you just went, and you didn't worry about anything. Now, they've got the different co-pays. It's weird, and if you don't know insurance, it's really weird." These claims likely exacerbated Gigi's low engagement and high levels of stigma.

A medical crisis can be stress-inducing and financially devastating, and nearly all participants in our study mentioned enduring economic challenges, including periods when they experienced homelessness. Participants noted that economic barriers and poor treatment reinforce structural racism within the medical model. High direct and indirect costs associated with medical care were cited repeatedly by participants. Eminem discussed the barriers that contributed to her moderate stress levels:

People can't afford that stuff where something happens where you do get kicked off. Who's paying it for you? I think all my three meds come to over $\$ 2,000$ and that's every month I get them. It's just the cost of meds is kind of outrageous, but what can we do?

The financial burden often exacerbates social, psychological, and economic barriers, meanwhile, the support to help patients circumvent these challenges is often minimal.

Structural barriers to care were also present within the clinic across models of treatment and service delivery. Participants with substance use issues were denied care unless they stopped using. Furthermore, racism and discrimination within the medical environment may lead to medical provider assumptions of drug-seeking. Topher expounds upon his medium-level stigma:

They don't believe you. They don't believe you, and yeah, that's what got me. I know you get all kinds of people that come through the door, but don't lump me with them. I had a $6 \mathrm{~mm}$ kidney stone and an 8 mm kidney stone, and his [the doctor's] words were, 'Why on earth do you still have these stones if they [a clinic] saw these over a year ago?' I said they told me I was just trying to get drugs, pain pills.

The geographic location of the HIV clinic was often described by participants as a barrier to care. Participants discussed the lack of privacy and feelings of shame and stigma, with the vast majority of people experiencing midlevel or higher stress and stigma. Furthermore, patient anonymity was often compromised both inside and outside of the clinic, likely contributing to low engagement, elevated levels of stress, and stigma. Even though Tony reported low stress and stigma, he discussed strategically scheduling his appointments for the earliest time available in the day so he can protect his privacy by going into the clinic when few others are there. Additionally, participants noted that simply being seen near the clinic can reveal a patient's status. For example, participant Bo noted moderate stress and stigma and spoke of the clinic location as a barrier to care:

If they see you coming out of there, or going in there, they know it's an HIV clinic there, and they automatically assume that that's what you've got, and you're going to get treatment or whatever, like up in clinic. Because you're in the hospital building, and the dentistry is right next to the clinic, and they see you're sitting where the clinic at, people that's going to the dentist; they see you sitting there-'Man, he must have AIDS.'

Community Barriers to Care Community barriers include economic, housing, and food insecurity. Many participants described difficulty in obtaining transportation due to the inefficiency, unreliability, and cost of public transportation. "Fox" reported low engagement, medium stigma, and high levels of stress, and unsurprisingly stated: "transportation is a big problem." HIV clinics often supplied bus tokens but failed to grasp the full scope of the problem. As "Junior" explained, "I come here and get tickets, but I run out because I've been using them to try to find an apartment. I can't get no more till next month." Even though these challenges, Junior maintained high engagement, low stigma, however, his stress levels were high-a likely byproduct of economic challenges. Gigi elaborated upon her high levels of stigma by explaining ongoing transportation barriers: 
"One bus, and I have to transfer. Initially, back when I was carrying oxygen tanks and stuff, it was hard to catch a bus because I get out in the heat, and the little tanks are only for two hours."

Participant experiences illustrate that simply getting bus tickets from community agencies is often insufficient and at times reinforces structural racism. Beyond the cost of bus fare, traveling by bus is slow, adversely affecting participants' ability to make it to their appointments on time. This reality was sometimes overlooked by clinic staff and medical providers. "Peter" stated that "the prejudices I have received is primarily due to my skin color versus my medical condition...There are a lot of things associated with being African American in this country. I think that has more prejudices than having HIV." His experiences directly relate to his survey data reports of high stress and medium stigma.

\section{Individual Barriers to Care}

Most participants faced health obstacles beyond living with HIV, including medication side effects, health comorbidities, substance use, lack of access to other health agencies, and generally poor medical care. Jethro described months of ongoing illness before finally being tested for HIV, meanwhile, he still endures high stress and stigma. Participants also described a snowball effect with health concerns, as one problem seemingly led to the next. All were coping with cooccurring conditions, such as hepatitis, cancer, pancreatitis, kidney disease, follicular lymphoma, diabetes and diabetic neuropathy, hypertension, chronic obstructive pulmonary disease, arthritis, boils, amblyopia, and dental issues. "Tee" had high engagement even with substantial medical and economic concerns likely affecting his medium stress and stigma levels:

I had all these responsibilities. Rent, traveling back and forth to all the doctor's appointments, and all of that stuff. I make \$991 disability. I pay $\$ 572$ for rent each month, all the utilities included. $\$ 100$ for transportation each month back and forth for dialysis. That's not including other expenses.

Gigi poignantly expressed the dilemma of keeping up with HIV treatment protocols when in the grip of substance use:

I was trying to take the medicine, but when you are on drugs you can't really be as compliant as you would if you were in your right mind. They want you to take it at the right time, eat right, try to exercise, make all your doctor's appointments. When you are on drugs, you just don't do that, even on my best days.
Barriers to care often merge across clinic, community, and individual levels. For example, Rabbit's ongoing health challenges exacerbated barriers to care across multiple levels. Specifically, she stated that staff turnover and the high number of interns at the local Veteran's Affairs office negatively affected the quality of care and her trust in medical providers: "The head man stays the same, but we can't see him, because that's what his interns are for. As far as actually sitting down with him, doing everything? No, his interns do that." Finally, participants disclosed struggles with stigma over their HIV status. Due to fear of negative reactions, "Roc" has told no one about his diagnosis, and how keeping this secret created a sense of loneliness.

Food insecurity was also noted by participants in the study. Economic insecurity often manifested additional barriers including insufficient food access and supply. Daphne alluded to these challenges stating, "My biggest problem now is even though I'm trying to get disability, I'm still trying to find some type of part-time work I can do because it's hard sometimes, just getting food stamps." At multiple points during the interview, participant, Tee underscored the difficulties of food insecurity and lack of support: "They don't give you that much food stamps. I literally get $\$ 18$ worth of food stamps every month." Finally, Peter underscored the importance of access to quality food to conjoin ongoing HIV treatment, in addition to stating that his financial challenges were not unique and that many seniors were in similar positions of financial hardship and food insecurity:

Because I worked all my life when I had HIV, I get a substantial, more than the average person gets on disability but that eliminates me from all other types of help and that's unfair. I cannot get food stamps but yet I have to pay the same price that everybody else does.

Additionally, Peter discussed the importance of quality food access:

If I have \$200 to spend for food, I cannot eat anything. I cannot eat Ramen noodles. I have to eat a balanced diet, not only because of cancer but because of HIV and the meds I take. I cannot eat pizza, if I could go get a box case of Ramen noodles and then I can live but I cannot.

Collectively, these findings reinforce the need for HIV care providers to become more aware of individual patients' experiences in order to reduce structural barriers, improve clinical support, and bolster quality care. Too often, economic challenges, including food, housing, and job insecurity, are viewed as entirely separate challenges facing patients, however, these experiences play an integral to the overall patient experience and directly influence HIV service provision. 


\section{Discussion}

Participants in our study reported experiences of inclusive HIV care where their voices were heard and their opinions represented, and where they felt valued, respected, cared for, and safe. Participants also consistently identified barriers to care and described how HIV care providers often fail to grasp the overwhelming nature of these barriers and the subsequent impact on their engagement and care [13].

Consistent with the literature on patient-centered care, all the participant experiences of quality care were rooted in an empathetic medical model oriented toward individualized, patient-centered care [14, 35]. Furthermore, quality care was directly predictive of future engagement in care, yet patient experiences of compassionate-based services were often few and far between, with positive experiences surpassed by substantial levels of stress and stigma. With every interaction, staff and clinicians have an opportunity to engage in considerate behavior and kind conversation, providing patients with real and symbolic support that directly affects patients' experiences and the likelihood that they will remain and thrive in care [9, 31-40]. Regardless of the predominance of high stress and stigma reported by participants, they maintained recognition of quality care and deeply appreciated every supportive and compassionate interaction.

Health comorbidities and medication side effects presented an array of challenges for participants in our study [22]. The wide-ranging health comorbidities presented barriers to care that was confounded by lack of provider understanding, diminished patient voice, likely contributing to elevated scores of stress and stigma for our study participants. Participants were, at times, perceived as drug-seeking when suffering from severe ailments. Critical race theory helps us uncover the pervasive role of racism, including the historically prejudicial notions that African Americans have higher levels of pain tolerance and are more prone to deception $[12,13,19,20]$. While these notions are false, the perpetuation of these beliefs leads to implicit racial biases that negatively impact HIV care [13, 14, 52]. Furthermore, the whiteness of healthcare professionals adds barriers; participants in this study often wished they had healthcare providers with shared identities (i.e., socioeconomic; race; ability; HIV positive) who could better understand their needs and experiences [42, 52]. Additionally, providers were often altogether dismissive of the patient voice, which worsened already poor care and reinforced racism and discrimination $[39,40]$. These stigmatizing beliefs held by medical providers perpetuate the high levels of internalized stigma felt by people living with HIV, noted often by the older African American study participants [26-29]. Additionally, the intersectional nature of multiple historically marginalized identities, including race, ethnicity, gender, and socioeconomic status, creates layers of discrimination and stigma, often internalized by patients $[10,13]$. Enhancing the patient's voice and attending to their experiences may help ameliorate some of these barriers to care by reducing structural forms of racism and ultimately aligning the medical care model to better address individual patient needs [9, 29-40].

Consistent with other studies, participants detailed an array of systemic barriers to care at the clinic, community, and individual level $[9,38]$. Most participants consistently dealt with economic challenges, including housing, food, and job insecurity. All of these barriers are more prevalent in urban communities, where food deserts are more common and employment opportunities are scarce [5]. Additional barriers include the difficulties met in attempting to manage the local bus system, and this hardship was often increased by HIV care providers' minimal understanding of the problems. Some HIV care providers recognized that poor transportation was a barrier to care but appeared to believe that supplying patients with bus tickets would suffice. Our findings illuminate the ways in which the privileged positionality of HIV care providers may negatively impact their ability to provide quality care [13].

Participants in our study showed widespread awareness and a deep appreciation for quality care that helped them feel more adept, empowered, and inspired to continue care. A positive relationship with medical providers directly facilitated participants' continued engagement [10, 11]. Consistent with the literature, reports of feeling heard and valued as a person directly impacted high levels of participant engagement in HIV care [39, 40]. Conversely, when participants felt discriminated against or received poor care, they felt devalued, misunderstood, and that their needs were being neglected by medical providers too busy to provide individualized and attentive care [26-28]. This study reinforces previous findings that healthy provider-patient relationships are a key driver of quality care, ultimately improving engagement of care for an older African American population [9, 37, 38].

\section{Implications}

Our findings show that barriers to care are widespread at the clinic, community, and individual levels, often leading to a lack of patient voice. These barriers are consistently disruptive at each stage of the HIV care continuum, hindering the ability of older African American patients to move fluidly through the medical system. The medical model continues to emphasize the importance of the individual patient adhering to care without considering barriers that disproportionately affect people of color [53-58]. Participants in our study discussed their ability to maneuver through barriers to care at the clinic, community, and individual levels while simultaneously demonstrating a keen awareness of the lack 
of inclusivity, patient voice, and unempathetic doctors that result in the poor care too often delivered by the top-down medical model [9, 37, 50]. Older African American participants in our study persevered through significant levels of stress and stigma, despite ongoing economic challenges, however, they were clear about the need for financial, community-based, and clinical support, including empathic, caring, and patient-centered care. While providers may be cognizant of barriers to care, it is unclear how well they pay attention to and value the concerns voiced by patients $[8,11$, 16]. Patients offer a window into their world, yet too often, their experiences are not recognized or respected within the top-down approach to medical care.

\section{Recommendations}

To bolster the patient voice throughout HIV care, we offer several recommendations for practice and policy designed to reduce disparities and improve service delivery for an older African American population. Our first recommendation for practice entails centralizing the patient voice in HIV service provision. Because patient experiences vary widely across identities and ideologies, care may improve through compassionate, patient-centered approaches that are specific to the individual. This can be achieved by incorporating a reinvigorated medical approach that values and emphasizes the lived experiences of an older African American population living with HIV.

Our second recommendation for practice is for HIV providers to identify barriers to care specific to an older African American population and as articulated by individual patient transgressions. Our sample talked at length about the importance of community-based services and support. One mechanism to bolster this support may be to enhance linkages among care services, recruit direct community partners, minimize referral services, and bolster support services for patients [43]. This recommendation is a direct extension of centralizing the patient voice, whereby linkage to community-based support services extends from unique patient understanding. Furthermore, providers may consider surveys and/or enhanced discussions with patients in order to amplify the patient voice and support older African American people.

Our first recommendation for policy is a redesign of the HIV care continuum to adequately account for the realities of patients' lived experiences. Currently, our sample population of older African Americans and their perspectives are absent from the HIV continuum. Consistent with previous findings, our research makes evident the barriers to care that upend patients' ability to move fluidly through the continuum and can cause them to fall out of care $[9,37$, $38,53-57]$. In alignment with study participant concerns and to bolster engagement in care, the HIV care continuum must address barriers to care, help reduce stress and stigma, improve service delivery, and enhance health outcomes. A reinvigorated HIV care continuum may help to redress systemic and structural barriers that undermine care and help to strengthen the older African American patient voice throughout their ongoing stay in care.

Our second recommendation for policy is to address systemic racism more generally for older African Americans, including explorations across barriers by way of economic, housing, food, and transportation [14]. Ryan White HIV/ AIDS Program funding exists to help address some of these needs and has been shown to increase retention in care; however, not all HIV care providers offer funding, and the breadth of funding is often limited in scope $[58,59]$. More funding is needed, in addition to providing enhanced accessibility to funds for HIV service providers. Finally, less is known about how Ryan White provides quality support for an older African American population.

\section{Limitations}

This study has several limitations. Participants were recruited from a limited geographical area that happens to be a city with racial segregation due to historical redlining practices and limited public transportation. Recruitment strategies, combined with small sample size, limit the generalizability of these findings. Most of the participants were between the ages of 50 and 65 years; an older sample population might yield new findings. Additionally, data were self-reported, and items related to engagement in care (e.g., number of missed clinic visits, diagnoses, and CD4 counts) were not based on medical chart review. Recall bias and social desirability bias may have influenced responses to the surveys.

\section{Conclusion}

Empathetic, patient-centered, relationship-driven models of HIV care were consistently identified as important to participants in our study and as directly facilitating their future engagement and retention in care. Supportive HIV service provision may offer a path toward uprooting structural barriers to care through a patient-centered response that values each individual experience. Quality HIV care can be achieved by centering the patient voice, understanding patients' experiences, and implementing care designed to reduce barriers and better meet individual patient needs.

Supplementary Information The online version contains supplementary material available at https://doi.org/10.1007/s40615-022-01237-2. 
Acknowledgements The authors of this study would like to make a special acknowledgment to the editor-in-chief, Dr. Cato Laurencin, and the anonymous reviewers of this study. Collectively, the input was very supportive, insightful, useful, and was thoroughly utilized to improve some important core concepts throughout this manuscript. We thank them graciously for their wonderful contributions.

Author Contribution Brandon Mitchell: research design, coding, analysis, writing, and editing; Liz Utterback: data coding, analysis, and writing; Paul Hibbeler: data coding; Ashley Logsdon: data coding; Patricia Smith: data coding; Lesley M. Harris: procurement of research funding, research design, data collection, writing, and editing; Billie Castle: research design and editing; Jelani Kerr: procurement of research funding, design conceptualization, data collection, and editing; Tim Crawford: procurement of research funding, design conceptualization, data collection, and editing.

Funding The authors would like to thank the University of Louisville's Vice President for Research and Innovation's Internal Research Grant Program for funding this research.

Data Availability Not applicable.

Code Availability Not applicable.

\section{Declarations}

Ethics Approval University of Louisville IRB Approval No. 20.0792.

Conflict of Interest The authors declare no competing interests.

\section{References}

1. Kalidasan V, Theva DK. Lessons learned from failures and success stories of HIV breakthroughs: are we getting closer to an HIV cure? Microbiol. 2020;11:46. https://doi.org/10.3389/fmicb.2020. 00046.

2. Centers for Disease Control and Prevention (CDC). HIV and older Americans. 2019. https://www.cdc.gov/hiv/group/age/olderameri cans/index.html. Accessed 1 June 2021.

3. Youssef E, Cooper V, Delpech V, Davies K, Wright J. Barriers and facilitators to HIV testing in people age 50 and above: a systematic review. Clin Med (Lond). 2017;17(6):508. https://doi.org/10.7861/ clinmedicine.17-6-508.

4. Centers for Disease Control and Prevention. Estimated HIV incidence and prevalence in the United States, 2014-2018. HIV Surveillance Report. 2020;25(1). https://www.cdc.gov/hiv/pdf/libra ry/reports/surveillance/cdc-hiv-surveillance-supplemental-reportvol-25-1.pdf. Accessed 1 June 2021.

5. Nocella AJ, Ducre KA, Lupinacci JL, editors. Addressing environmental and food justice toward dismantling the school-to-prison pipeline. London: Palgrave-MacMillan; 2017.

6. Van Slyke A. America's legacy of redlining: state-sponsored segregation and disenfranchisement of urban minority communities. Lerner Center for Public Health Promotion. Syracuse, NY: Syracuse University; 2020.

7. Denning P, DiNenno E. Communities in crisis: is there a generalized HIV epidemic in impoverished urban areas of the United States? XVIII international AIDS conference. Vienna, Austria; 2010 .
8. Dombrowski JC, Simoni JM, Katz DA, Golden MR. Barriers to HIV care and treatment among participants in a public health HIV care relinkage program. AIDS Patient Care STDS. 2015;29(5):279-87. https://doi.org/10.1089/apc.2014.0346.

9. Sangaramoorthy T, Jamison A, Dyer T. Older African Americans and the HIV care continuum: a systematic review of the literature, 2003-2018. AIDS Behav. 2019;23(4):973-83. https:// doi.org/10.1007/s10461-018-2354-4.

10. Harris LM, Crawford TN, Kerr JC, Thomas TA, Schmidt V. African American older adults living with HIV: exploring stress, stigma and engagement in hiv care. J Health Care Poor Underserved. 2020;31(1):265-286; https://doi.org/10.1353/hpu. 2020.0022.

11. Tobias CR, Cunningham W, Cabral HD, et al. Living with HIV but without medical care: barriers to engagement. AIDS Patient Care STDS. 2007;21(6):426-34. https://doi.org/10.1089/apc. 2006.0138.

12. Chowkwanyun M, Reed AL Jr. Racial health disparities and Covid-19 caution and context. N Engl J Med. 2020;383(3):2013. https://doi.org/10.1056/NEJMp2012910.

13. Freeman R, Gwadz MV, Silverman E, et al. Critical race theory as a tool for understanding poor engagement along the HIV care continuum among African American/Black and Hispanic persons living with HIV in the United States: a qualitative exploration. Int J Equity Health. 2017;16(1):1-14. https://doi.org/10. 1186/s12939-017-0549-3.

14. Laurencin CT, Walker JM. A pandemic on a pandemic: racism and COVID-19 in Blacks. Cell Syst. 2020;11(1):9-10.

15. Collins PH. Learning from the outsider within: the sociological significance of black feminist thought. Soc Probl. 1986;33(6):s14-32.

16. Bradford JB, Coleman S, Cunningham W. HIV system navigation: an emerging model to improve HIV care access. AIDS Patient Care STDS. 2007;21(Suppl 1):S49-58. https://doi.org/ 10.1089/apc.2007.9987.

17. hooks b. Feminist theory: from margin to center. Philadelphia: 1984.

18. Kendall M. Hood feminism: notes from the women that a movement forgot. New York: Penguin; 2021.

19. Taylor E, Gillborn D, Ladson-Billings G. Foundations of critical race theory in education. Oxfordshire: Routledge; 2009.

20. Delgado R, Stefancic J. Critical race theory: the cutting edge. Philadelphia: Temple University Press; 2013.

21. Byrd KK, Hardnett F, Clay PG, et al. Retention in HIV care among participants in the patient-centered HIV care model: a collaboration between community-based pharmacists and primary medical providers. AIDS Patient Care STDS. 2019;33(2):58-66. https://doi.org/10.1089/apc.2018.0216.

22. Byrd KK, Hardnett F, Hou JG, et al. Improvements in retention in care and HIV viral suppression among persons with HIV and comorbid mental health conditions: patient-centered HIV care model. AIDS Behav. 2020;24:3522-32.

23. Jaiswal J, Griffin-Tomas M, Singer SN, Lekas HM. Desire for patient-centered HIV care among inconsistently engaged racial and ethnic minority people living with HIV. J Assoc Nurses AIDS Care. 2018;29(3):426-38. https://doi.org/10.1016/j.jana. 2018.01.001.

24. Hawkins JM, Mitchell J. The doctor never listens: older African American men's perceptions of patient-provider communication. Soc Work Res. 2018;42(1):57-63. https://doi.org/10.3390/ geriatrics3030038.

25. Burchett CO, Shen MJ, Freeman R, et al. Using focus group feedback to identify patient-centered initiatives for older persons with HIV. Clin Gerontol. 2020:1-12. https://doi.org/10. 1080/07317115.2020.1769245 
26. Geter A, Herron AR, Sutton MY. HIV-related stigma by healthcare providers in the United States: a systematic review. AIDS Patient Care STDS. 2018;32(10):418-24. https://doi.org/10.1089/ apc.2018.0114.

27. Harris LM, Emlet CA, Pierpaoli Parker C, Furlotte C. Timing of diagnosis: understanding resilience narratives of HIV positive older adults diagnosed pre- and post-HAART. J Gerontol Soc Work. 2018;61(1):78-103. https://doi.org/10.1080/01634372. 2017.1402841.

28. Kerr J, Harris L, Glass E, Golden T, Crawford T. "I shall live and not die": using monologues based on the experiences of older African Americans living with HIV to address HIV-related stigma among African Americans in Louisville. Kentucky Fam Community Health. 2020;43(4):257-63. https://doi.org/10.1097/FCH. 0000000000000268 .

29. Miller Jr, WL. Experiences of stigma and spirituality of older Black men living with HIV. J Soc Serv Res. 2019

30. Beane SN, Culyba RJ, DeMayo M, Armstrong W. Exploring the medical home in Ryan White HIV care settings: a pilot study. J Assoc Nurses AIDS Care. 2014;25(3):191-202.

31. Trzeciak S, Mazzarelli A, Booker C. Compassionomics: the revolutionary scientific evidence that caring makes a difference. Pensacola, FL: Studer Group. 2019

32. Mizuno Y, Higa DH, Leighton CA, Roland KB, Deluca JB, Koenig LJ. Is HIV patient navigation associated with HIV care continuum outcomes? AIDS (London, England). 2018;32(17):2557.

33. Mizuno Y, Higa DH, Leighton CA, Roland KB, Deluca JB, Koenig LJ. Is HIV patient navigation associated with HIV care continuum outcomes? AIDS. 2018;32(17):2557-71. https://doi. org/10.1097/QAD.0000000000001987.

34. Roland KB, Higa DH, Leighton CA, Mizuno Y, DeLuca JB, Koenig LJ. Client perspectives and experiences with HIV patient navigation in the United States: a qualitative meta-synthesis. Health Promot Pract. 2020;21(1):25-36.

35. Christopoulos KA, Massey AD, Lopez AM, et al. "Taking a half day at a time": patient perspectives and the HIV engagement in care continuum. AIDS Patient Care STDS. 2013;27(4):223-30. https://doi.org/10.1089/apc.2012.0418.

36. Land L, Hathorn E, Ross JDC. Using patient experience to measure the quality of HIV care. Int J STD AIDS. 2011;22(7):366-7.

37. Sangaramoorthy $\mathrm{T}$, Jamison A, Dyer T. Intersectional stigma among midlife and older Black women living with HIV. Cult Health Sex. 2017;19(12):1329-43.

38. Emlet CA, Harris L. Giving back is receiving: the role of generativity in successful aging among HIV-positive older adults. J Aging Health. 2020;32(1-2):61-70.

39. Beach MC, Keruly J, Moore RD. Is the quality of the patientprovider relationship associated with better adherence and health outcomes for patients with HIV? J Gen Intern Med. 2006;21(6):661-5.

40. Flickinger TE, Saha S, Roter D, Korthuis PT, Sharp V, Cohn J, ... Beach MC. Clinician empathy is associated with differences in patient-clinician communication behaviors and higher medication self-efficacy in HIV care. Patient education and counseling. 2016;99(2):220-226

41. Hassen N, Lofters A, Michael S, Mall A, Pinto AD, Rackal J. Implementing anti-racism interventions in healthcare settings: a scoping review. Int J Environ Res Public Health. 2021;18(6):2993.

42. Yearby R. Racism is a public health crisis. Doctoral dissertation, Saint Louis University. 2020

43. Safreed-Harmon K, Anderson J, Azzopardi-Muscat N, Behrens GM, Monforte ADA, Davidovich U, ... Lazarus JV (2019)
Reorienting health systems to care for people with HIV beyond viral suppression. The Lancet HIV;6(12):e869-e877.

44. Creswell JW, Plano Clark VL. Designing and conducting mixed methods research. 2nd ed. London: Sage Publications Ltd.; 2011.

45. Charmaz K. The power of constructivist grounded theory for critical inquiry. Qual Inq. 2017;23(1):34-45.

46. Blumer H. Symbolic interactionism: perspective and method. Oakland: University of California Press; 1986.

47. Mead GH. Mind, self and society. Chicago: University of Chicago Press; 1934.

48. Berger BE, Ferrans CE, Lashley FR. Measuring stigma in people with HIV: psychometric assessment of the HIV stigma scale. Res Nurs Health. 2001;24(6):518-29. https://doi.org/10.1002/nur. 10011.

49. Cohen S, Williamson G. Perceived stress in a probability sample of the US. In: Spacapam S, Oskamp S, editors. The social psychology of health: the Claremont symposium on applied social psychology. Newbury Park, CA: Sage Publications; 1988. p. 31-67.

50. Saberi P, Johnson MO. Moving toward a novel and comprehensive behavioral composite of engagement in HIV care. AIDS Care. 2015;27(5):660-4. https://doi.org/10.1080/09540121.2014. 986052 .

51. Miles MB, Huberman AM, Saldana J. Qualitative data analysis: a methods sourcebook. 2014

52. Castle B, Wendel M, Kerr J, Brooms D, Rollins A. Public health's approach to systemic racism: a systematic literature review. J Racial Ethn Health Disparities. 2019;6(1):27-36.

53. Kay ES, Batey DS, Mugavero MJ. The HIV treatment cascade and care continuum: updates, goals, and recommendations for the future. AIDS Res Ther. 2016;13(1):1-7. https://doi.org/10.1186/ s12981-016-0120-0.

54. Jose S, Delpech V, Howarth A, et al. A continuum of HIV care describing mortality and loss to follow-up: a longitudinal cohort study. Lancet HIV. 2018;5(6):e301-8. https://doi.org/10.1016/ S2352-3018(18)30048-1.

55. Mugavero MJ. Elements of the HIV care continuum: improving engagement and retention in care. Top Antivir Med. 2016;24(3):115-9.

56. Ogden J, Esim S, Grown C. Expanding the care continuum for HIV/AIDS: bringing carers into focus. Health Policy Plan. 2006;21(5):333-42. https://doi.org/10.1093/heapol/czl025.

57. Barrington C, Davis DA, Villa-Torres L, Carcano J, HightowWeidman L. Intersectionalities and the HIV continuum of care among gay Latino men living with HIV in North Carolina. Ethn Health. 2019:1-16. https://doi.org/10.1080/13557858.2019.16201 77

58. Kay ES, Westfall AO. Ryan White HIV/AIDS program recipients more likely than non-recipients to be retained in care using six different retention measures. AIDS care. 2020;32(1):89-92; https:// doi.org/10.1080/09540121.2019.1623375

59. Cahill SR, Mayer KH, Boswell SL. The Ryan White HIV/AIDS program in the age of health care reform. Am J Public Health. 2015;105(6):1078-85. https://doi.org/10.2105/AJPH.2014. 302442 .

Publisher's Note Springer Nature remains neutral with regard to jurisdictional claims in published maps and institutional affiliations. 